\title{
Impacto del programa Generación E en la Educación Superior durante el COVID-19
}

\section{Impact of the Generation E program on Higher during COVID-19}

SUÁREZ CASTRILLÓN, Albert M. ${ }^{1}$

RINCÓN, Isbelia K. ${ }^{2}$

SUÁREZ, Sir A. ${ }^{3}$

\begin{abstract}
Resumen
El artículo presenta el impacto del programa Generación E en la Universidad Francisco de Paula Santander, Ocaña, iniciativa del gobierno para dar mayor equidad en la educación superior. Los beneficios antes y durante el COVID-19 han mostrado un rendimiento académico no condicionado del $93,39 \%$, y una cobertura entre el $66 \%$ y $78 \%$ entre los estudiantes de estratos 1, 2 y 3; razón por la cual se debe revisar el puntaje otorgado por el SISBEN a estudiantes fuera del programa.

Palabras clave: generación e, covid-19, cobertura educativa

Abstract

The impact of the Generation E program at the Francisco de Paula Santander Ocaña University, a government initiative to provide greater equity in higher education, is presented. The benefits before and during COVID-19 have shown an unconditional academic performance of $93.39 \%$. And a coverage between $66 \%$ and $78 \%$, among students of strata 1,2 and 3; reason why the score given by SISBEN to students outside the program should be reviewed.

key words: generation e, covid-19, educational coverage
\end{abstract}

\section{Introducción}

El Covid-19 es una enfermedad respiratoria que ha afectado a todos los sectores a nivel mundial, impactando con más fuerza en los países en vías de desarrollo, que de por sí, ya tenían problemas acumulados de crecimiento y deudas externas, obligándolos a un endeudamiento casi exprés; y según, cifras del Banco Mundial, para el año 2018 la deuda alcanzaba los 7,8 billones de dólares (BM, 2020). Sus síntomas más comunes son fiebre y tos, y es altamente contagiosa (Casas-Rojo et al., 2020), originando un cierre casi total de empresas, instituciones educativas, transporte, salud, entre otras. EI COVID-19 ha cambiado las actividades diarias de toda la población, obligando a tomar medidas de bioprotección, y convirtiendo a la mascarilla en uno de los objetos más confiables y económicos para la mitigación y protección individual (Aranaz et al., 2020). Colombia es uno de los países más afectados de Suramérica, con un porcentaje de contagios de 10,42\% que lo ubican en una segunda posición, y tercero en cuanto a personas fallecidas, con 10,22\% para principios de octubre de 2020 (Pan American Health Organization, PAHO, 2020). El gobierno nacional ha decretado diferentes medidas (MINSALUD, 2020a), que se

\footnotetext{
${ }^{1}$ Docente. Programa Ingenieria Mecánica. Universidad de Pamplona. Grupo de Investigación Gimup. albertmiyer@unipamplona.edu.co

2 Docente. Programa Ingenieria de Sistemas. Universidad Francisco de Paula Santander Ocaña. Grupo de Investigación Grucite. ikrinconp@ufpso.edu.co

${ }^{3}$ Docente. Programa Ingenieria de Sistemas. Universidad Francisco de Paula Santander Ocaña. Grupo de Investigación Grucite. sasuarezc@ufpso.edu.co
} 
siguen implementando con la finalidad de reducir los niveles de contagio y superar la cuarentena impuesta en cada uno de sus territorios desde el 12 de marzo de 2020 (MINSALUD, 2020b).

En el año 2019 Colombia presentó una reducción en la pobreza de $27 \%$ al 50\% entre los años 2002 y 2017, pero solo 36 de cada 100 personas tienen un trabajo regido por las medidas de Ley (Alonso, 2019), pero con el deterioro generado por la pandemia sale una nueva pregunta, ¿El impacto económico del COVID-19 abrirá nuevas brechas en la sociedad? De acuerdo con el Departamento Administrativo Nacional de Estadística, DANE (2020), 59,8\% de las personas cabeza de familia considera que la situación económica empeoró en el último año, según la encuesta de Pulso Social creada con la finalidad de conocer: la confianza del consumidor, el conocimiento de los apoyos sociales y el bienestar de las familias integradas por niños, niñas y adolescentes.

Esta disminución del nivel de ingresos por familia genera un impacto en la educación, el cual puede llevar a la deserción estudiantil y a la posibilidad de no ingresar a un ciclo formativo. Hablar de equidad en la educación es hablar de dificultades relacionadas al estrato socioeconómico, y sus deficiencias por cobertura, las cuales han llevado al país a estar en desventaja con otras naciones de Suramérica (Rojas, 2018). Ingresar a la educación superior parece ser más difícil si los recursos en la educación preescolar, básica y media no están bien enfocados, y las metodologías pueden ser cambiantes (Castro Archila, 2018) si se entiende que los resultados no están siendo los esperados. El acceso a la educación superior puede manifestarse por indicadores como: cobertura, cantidad de docentes y financiación de las instituciones; pero finalmente sus logros dependen de la condición socioeconómica (Melo-Becerra, Ramos-Forero y Hernández-Santamaría, 2017). La tendencia a la baja en la matrícula de las universidades ya era latente antes del COVID-19 con una reducción desde el año 2016, la cual puede aumentar en $12 \%$ para el segundo semestre de 2020. En Colombia, $45 \%$ de los jóvenes no finalizan sus estudios (ASCUN, 2020) y para el mes de mayo las cifras aumentaron su pronóstico en deserción con un $25 \%$ según Ascun (2020), que incluso podría extenderse hasta 2022.

El gobierno nacional ha tomado nuevas medidas para el apoyo al sector educativo, con la finalidad de evitar la deserción y aumentar el acceso a la educación superior, como son: créditos en condiciones especiales, destinando $\$ 2,5$ billones a través del Fondo Nacional de Garantías, el cual pretende cubrir $90 \%$ de la nómina, y un crédito de Findeter de $\$ 200.000$ millones para apoyar a los estudiantes de pregrado; también del Fondo Solidario para la Educación Superior a estudiantes de estratos 1 y 2 (Mineducación, 2020). Las medidas pueden aliviar las cargas económicas que implican cursar una carrera de educación superior, y al ser transitorias y de créditos, puede dejar una inseguridad en el núcleo familiar, y más, en el estudiante que no se sentirá del todo protegido para centrarse en su labor académica. Los programas de ayuda permanente pueden ser más efectivos en el rendimiento económico, los cuales ya estaban siendo ejecutados por el gobierno antes de la pandemia, ellos son: Jóvenes en Acción (JEA) y Generación E, los dos programas ayudan a la población en condición de pobreza y vulnerabilidad.

JEA es un programa para estudiantes entre los 16 y 28 años (Departamento para la Paz Social, DPS, 2020), mediante el cual se realizan dos entregas de ayuda por semestre. Para ingresar al programa, el estudiante ya debe encontrarse matriculado en una institución, mientras que Generación E otorga el beneficio de matrícula durante toda la carrera, y puede obtener el beneficio sin estar admitido por alguna institución. ¿Qué sería mejor para un estudiante? ¿Será más efectivo un programa de ayuda antes de iniciar la carrera? ¿Obtener la ayuda una vez este en la carrera?, en ese horizonte el JEP por ser un programa más antiguo ya ha demostrado algunos beneficios para los beneficiarios e inclusive a los docentes que imparten las clases (Carvajal, Vergel y Ortega, 2020). Pero la puesta en marcha del programa Generación E en el año 2018 admitió que necesitaba un complemento, que brindara seguridad con una ayuda adquirida con anterioridad, la cual permita al aspirante concentrarse en la elección de la carrera según sus aptitudes y deseos de aprender. El programa en su comienzo beneficiará a 336 mil estudiantes (Mineducación, 2018), y tiene los componentes de equidad y excelencia, el 
primer componente se da por méritos académicos y condición socioeconómica abarcando la mayor cantidad de estudiantes.

El objetivo de esta investigación es conocer el impacto del programa Generación E, antes y durante la pandemia del COVID-19, analizando indicadores de rendimiento académico, deserción e inclusión académica en la Universidad Francisco de Paula Santander Ocaña (UFPSO), la cual presenta indicadores socioeconómicos con estratos 1, 2 y 3 que la hacen susceptibles de ser una de las instituciones con mayor número de beneficiarios de acuerdo a los requisitos del programa. El análisis se realiza con una muestra de tres semestres desde el año 2019 hasta el tiempo del COVID-19.

\section{Metodología}

Se utiliza una metodología descriptiva, mediante la observación cuantitativa de los datos de estudiantes de la UFPSO, para los periodos 2019-1, 2019-2 y 2020-1, con una muestra tomada de 12 programas académicos y estudiantes beneficiados por el programa Generación E, cuyas métricas de medidas utilizadas son la cantidad de estudiantes con cobertura, rendimiento académico y el estrato social. El total es de 5.793 estudiantes, con una muestra de 1.452 estudiantes potenciales y 985 beneficiados, resaltando las variables de vulnerabilidad del programa en su componente de equidad para el periodo del COViD-19 semestre 2020-1 de 469 matriculados y 311 beneficiados.

El rendimiento académico se mide en base a tres estados: activo no condicionado con notas mayores o iguales a 3.1 , condicionado con notas menores a 3,1, y no activo para estudiantes con semestre cancelado o que desertaron. Y los estratos 1, 2 y 3 que tienen una vulnerabilidad alta (puntaje 0 y 54.86), media (puntaje 54.86 y 57.21) y baja (57.21 y 61.91), según los puntajes del SISBEN (Sistema de Identificación de Potenciales Beneficiarios de Programas Sociales) en que se encuentren (Clavijo, 2017).

Los datos se obtienen de los reportes de matrícula (R1) y permanencia y excelencia (R2) del proceso de Admisiones, Registro y Control de la UFPSO, ya condicionados para entrega al programa JEP y al Ministerio de Educación Nacional.

\section{Resultados}

Se realiza un análisis de cobertura del programa de generación basado en el estrato social, la cantidad de estudiantes matriculados vs beneficiados, la deserción estudiantil y rendimiento académico.

\subsection{Análisis del estrato social}

Para ingresar al programa de Generación E el aspirante o estudiante debe estar registrado en la base nacional del SISBEN y tener una clasificación igual o menor de 32 puntos. La UFPSO cuenta con cuatro facultades e imparte 12 programas académicos, de los cuales dos son nocturnos. Y los estratos económicos de sus estudiantes son de los más bajos en Colombia, como se puede ver en el cuadro 1.

Los datos reflejan que si los estratos 1, 2 y 3 son los de mayor vulnerabilidad, la UFPSO presenta un $99 \%$ en este rango y estarían condicionados todos los estudiantes que ingresan a ser beneficiarios del programa, y casi seguro más de $97 \%$ por pertenecer a los estratos 1 y 2 , tal como se muestra en el gráfico 1. 
Cuadro 1

Estrato social estudiantes matriculados 2020-1

\begin{tabular}{|c|c|c|c|c|c|c|c|}
\hline \multirow[t]{2}{*}{ Programa Académico } & \multicolumn{6}{|c|}{ Porcentaje estrato social } & \multirow{2}{*}{$\begin{array}{l}\text { Total } \\
\text { estudiantes }\end{array}$} \\
\hline & 1 & 2 & 3 & 4 & 5 & 6 & \\
\hline Ingeniería Ambiental & 75.91 & 22.85 & 1.63 & 0 & 0 & 0.18 & 552 \\
\hline Ingeniería Civil & 73.86 & 23.86 & 2.11 & 0.16 & 0 & 0 & 616 \\
\hline Ingeniería Mecánica & 80.48 & 19.08 & 0.44 & 0 & 0 & 0 & 456 \\
\hline Ingeniería de Sistemas & 77.13 & 21.08 & 1.79 & 0 & 0 & 0 & 446 \\
\hline Contaduría Pública diurna & 85.56 & 14.08 & 0.18 & 0.18 & 0 & 0 & 568 \\
\hline Contaduría Pública nocturna & 79.17 & 20.31 & 0.52 & 0 & 0 & 0 & 192 \\
\hline Derecho diurno & 71.45 & 25.41 & 2.8 & 0.23 & 0 & 0.12 & 858 \\
\hline Derecho nocturno & 65.11 & 29.67 & 5.22 & 0 & 0 & 0 & 364 \\
\hline Comunicación Social & 74.44 & 23.49 & 2.07 & 0 & 0 & 0 & 579 \\
\hline Zootecnia & 77.72 & 19.63 & 2.39 & 0.27 & 0 & 0 & 377 \\
\hline TEC. Gestión Comercial y financiera & 72.55 & 25.10 & 2.35 & 0 & 0 & 0 & 255 \\
\hline Administración de Empresas & 75.85 & 22.45 & 1.7 & 0 & 0 & 0 & 530 \\
\hline
\end{tabular}

Fuente: elaboración propia

Gráfico 1

Porcentaje de estudiantes condicionados para el programa

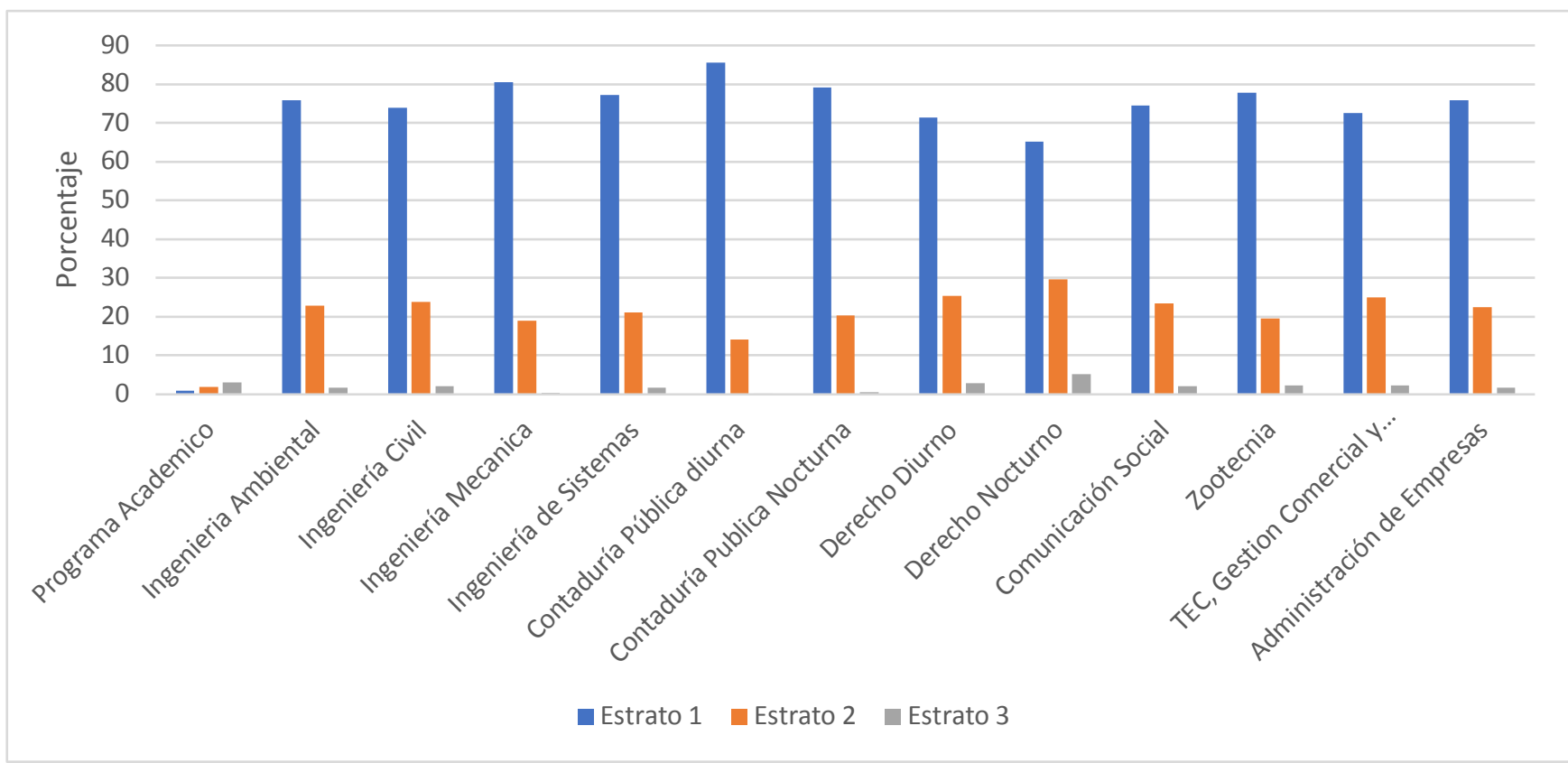

Fuente: elaboración propia

\subsection{Equidad y cobertura educativa generada por Generación $E$}

El ingreso a la universidad para los periodos 2019-1, 2019-2 y durante la pandemia 2020-1 COVID-19 se muestra en el cuadro 2, reflejando que la pandemia no ha sido trascendental para el aumento de la cobertura en los diferentes programas de la UFPSO. La tendencia de ingreso de estudiantes se mantiene e incluso en programas como: Ingeniería Mecánica, Contaduría Pública nocturna, Derecho diurno y nocturno, Comunicación Social y Tecnología en Gestión Comercial Financiera han decrecido un 50\%, como resultado, el impacto de Generación $\mathrm{E}$ no genera mayor aumento en la cobertura por parte de los comités curriculares de la UFPSO. 
Cuadro 2

Estudiantes matriculados antes y durante el COVID-19

\begin{tabular}{|l|c|c|c|c|}
\hline \multicolumn{1}{|c|}{$\begin{array}{c}\text { Programa } \\
\text { Académico }\end{array}$} & $2019-1$ & $2019-2$ & $2020-1$ & $\begin{array}{c}\text { Cantidad } \\
\text { estudiantes }\end{array}$ \\
\hline Ingeniería Ambiental & 39 & 35 & 42 & 116 \\
\hline Ingeniería Civil & 36 & 35 & 43 & 114 \\
\hline Ingeniería Mecánica & 37 & 35 & 36 & 108 \\
\hline Ingeniería de Sistemas & 43 & 35 & 47 & 125 \\
\hline Contaduría Pública diurna & 36 & 40 & 40 & 116 \\
\hline Contaduría Pública nocturna & 38 & 40 & 34 & 112 \\
\hline Derecho diurno & 38 & 35 & 34 & 107 \\
\hline Derecho nocturno & 33 & 35 & 30 & 98 \\
\hline Comunicación Social & 34 & 35 & 31 & 100 \\
\hline Zootecnia & 35 & 35 & 41 & 111 \\
\hline Tec. Gestión Comercial y financiera & 86 & 80 & 36 & 202 \\
\hline Administración de Empresas & 53 & 35 & 55 & 143 \\
\hline
\end{tabular}

Fuente: elaboración propia

En el cuadro 3, se muestra la cantidad de beneficiados por el programa Generación E para los tres semestres, dejando ver que no todos los estudiantes de los estratos 1, 2 y 3 logran ingresar al programa, demostrando que hace falta una revisión del puntaje que aparece en el SISBEN, en numerosas ocasiones los estudiantes han manifestado al proceso de Admisiones, Registro y Control, sus deficiencias básicas para continuar con el estudio y no lograr ingresar al programa, con casos donde los aspirantes no cuentan con servicio eléctrico y mucho menos con acceso a internet; por consiguiente genera dudas el puntaje registrado en materia socioeconómica.

\section{Cuadro 3}

Beneficiarios Generación E

antes y durante el COVID-19

\begin{tabular}{|l|c|c|c|c|}
\hline \multicolumn{1}{|c|}{ Programa Académico } & $2019-1$ & $2019-2$ & $2020-1$ & Cantidad estudiantes \\
\hline Ingeniería Ambiental & 29 & 28 & 26 & 83 \\
\hline Ingeniería Civil & 40 & 32 & 34 & 106 \\
\hline Ingeniería Mecánica & 24 & 18 & 25 & 67 \\
\hline Ingeniería de Sistemas & 36 & 30 & 27 & 93 \\
\hline Contaduría Pública diurna & 34 & 35 & 27 & 96 \\
\hline Contaduría Pública nocturna & 14 & 15 & 15 & 44 \\
\hline Derecho diurno & 35 & 40 & 28 & 103 \\
\hline Derecho nocturno & 11 & 10 & 8 & 29 \\
\hline Comunicación Social & 28 & 27 & 24 & 79 \\
\hline Zootecnia & 23 & 22 & 26 & 71 \\
\hline Tec. Gestión Comercial y financiera & 45 & 30 & 29 & 104 \\
\hline Administración de Empresas & 38 & 30 & 42 & 110 \\
\hline
\end{tabular}

Fuente: elaboración propia

En el gráfico 2, se puede observar la cobertura del programa para el periodo 2019-1, año en que se inició en la UFPSO. Logrando una cobertura aceptable en muchos programas, resaltando Ingeniería Civil, Contaduría pública día y Derecho diurno, donde todos los aspirantes lograron ser seleccionados, y ubicándose en un porcentaje bajo en la carrera de Tecnología en Gestión Comercial y Financiera. 
Gráfico 2

Cantidad estudiantes

matriculados vs beneficiados 2019-1

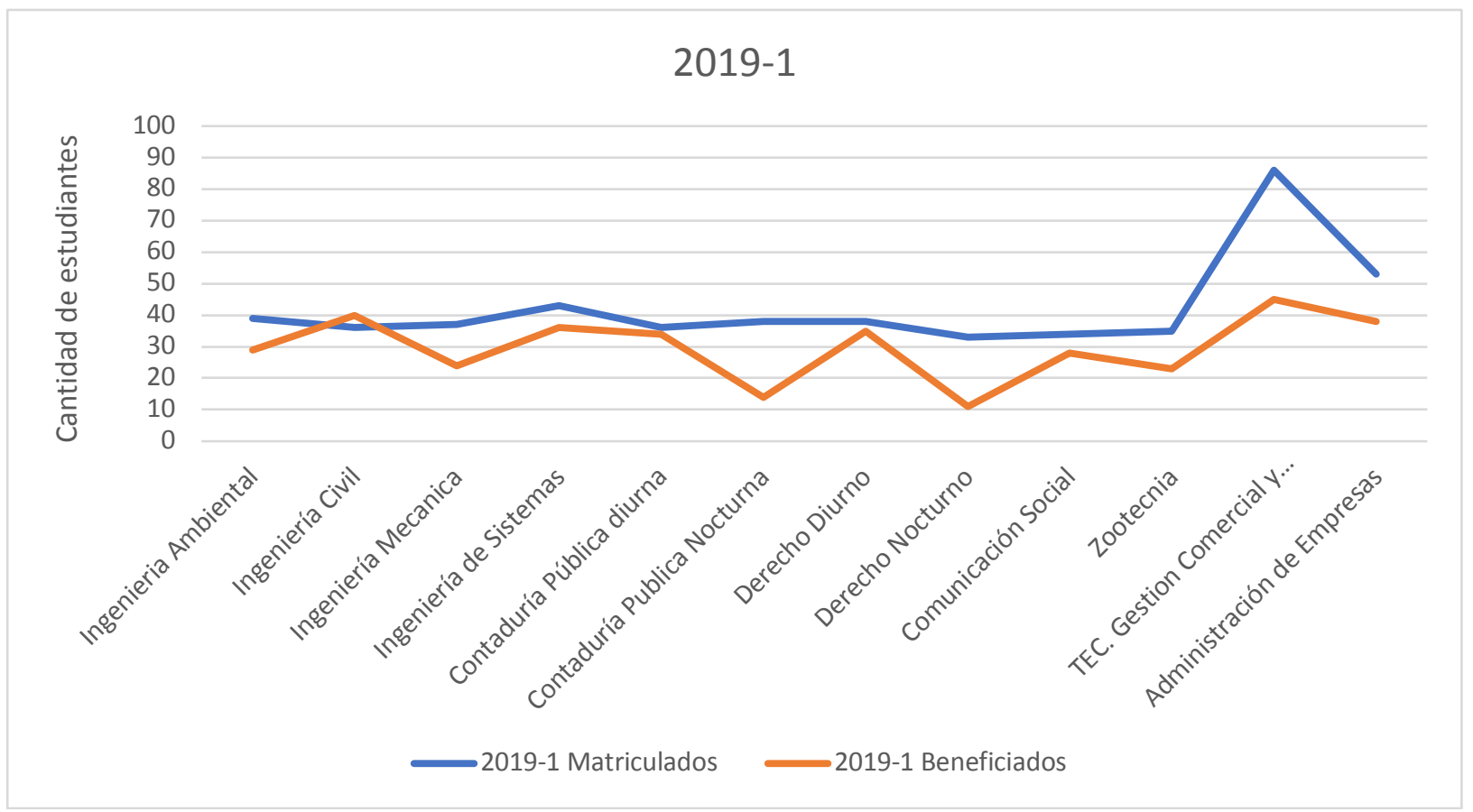

Fuente: elaboración propia

Para el periodo 2019-2, la tendencia de cobertura se sigue manteniendo en todos los programas (ver gráfico 3), aumentando su cobertura en Administración de Empresas; y se observa el caso del programa Derecho nocturno donde la cobertura no es la deseada y se debe al requisito de edad el cual llega hasta los 28 años.

El gráfico 4 es quizás el más importante al hablar del COVID-19, debido a que los resultados llegaron a mitad de año de la pandemia, y las cifras no muestran una tendencia de cobertura esperada, inclusive alejándose de los porcentajes de los años anteriores, si bien es cierto que el gobierno ha aumentado la cantidad de recursos para el programa, también es cierto que se debe a las normativas del sistema para revisar los criterios de cada estudiante. Sin embargo, es de resaltar que el Programa Generación E envía constantemente listados de nuevos admitidos, con un periodo comprendido entre el primer y tercer semestre, así queda una esperanza para cubrir a los estudiantes que faltan por ingresar. En muchas ocasiones se debe a que el estudiante no envía a tiempo el formulario de inscripción; el formulario es relativamente sencillo de diligenciar, debido a que todo se hace vía web y no amerita gastos económicos o de transporte. Como oportunidad de mejora, la UFPSO envía constantemente correos a todos los estudiantes que ingresan para que diligencien la inscripción y no queden por fuera de los beneficios del gobierno. 
Gráfico 3

Cantidad estudiantes

matriculados vs beneficiados 2019-2

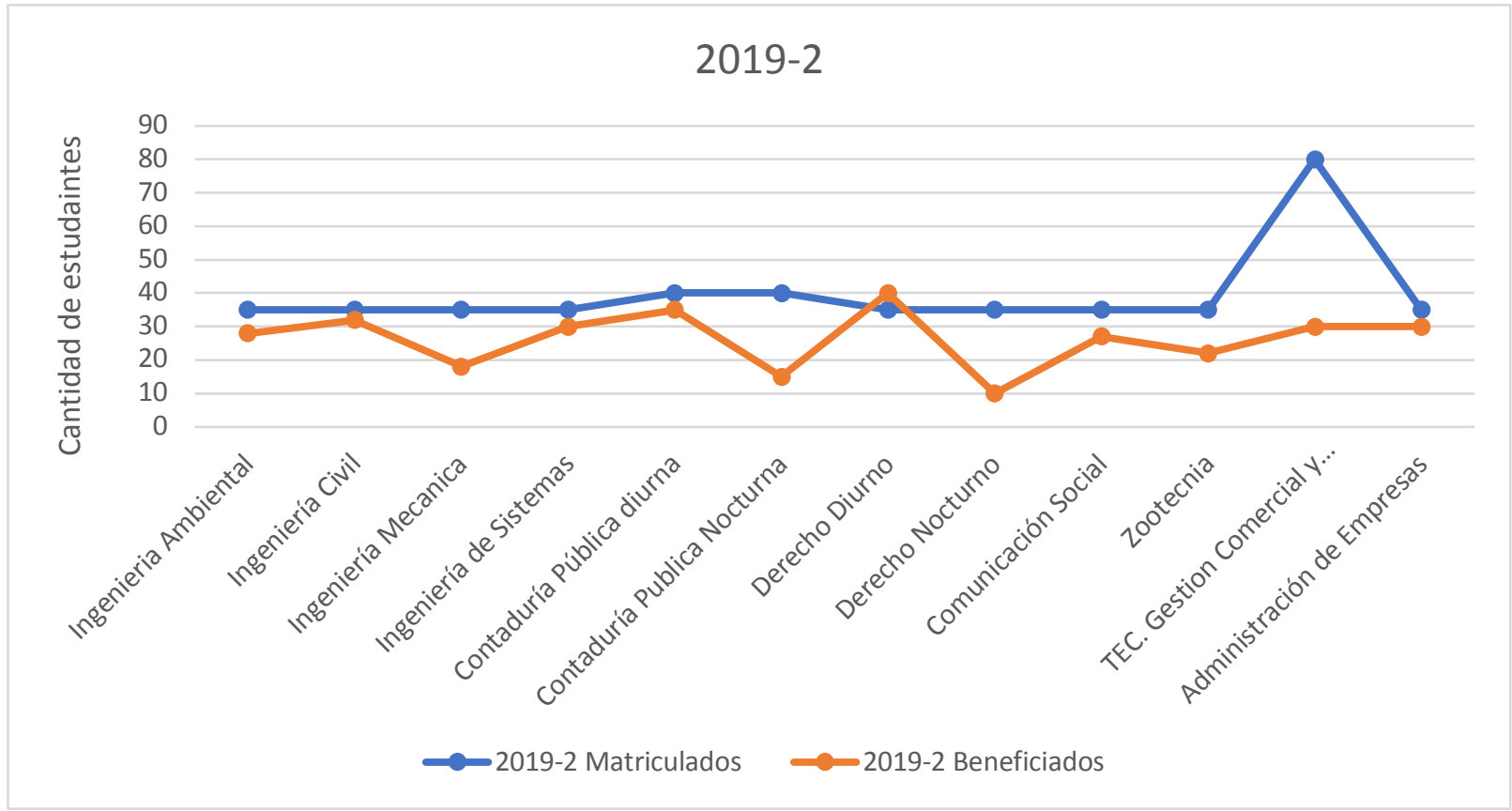

Fuente: elaboración propia

Gráfico 4

Cantidad estudiantes

matriculados vs beneficiados en tiempo de COVID-19

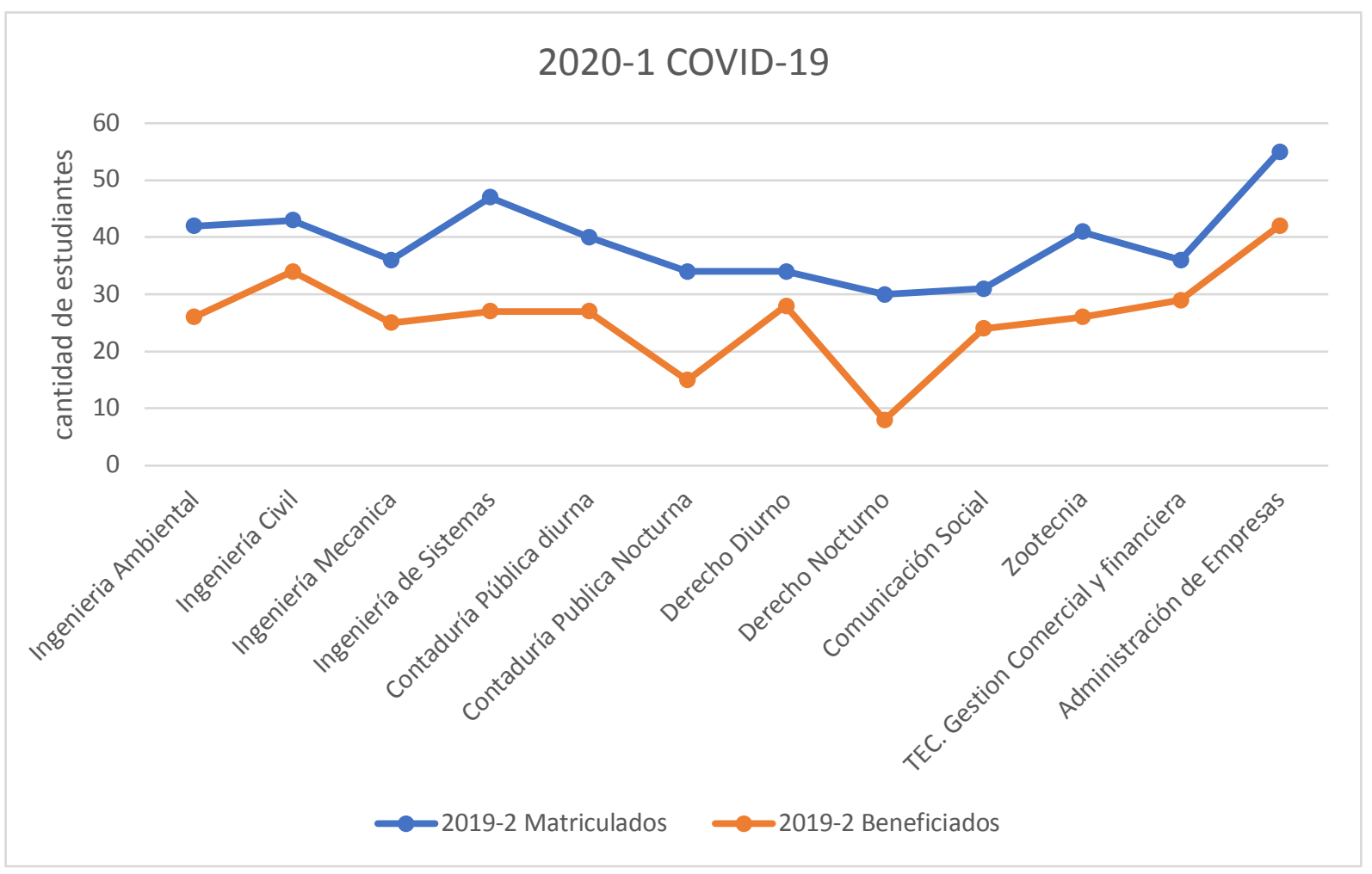

Fuente: elaboración propia 
El porcentaje total de cobertura que se refleja en el Gráfico 5 muestra la cantidad total de beneficiados vs matriculados, con un $70,27 \%$ de cobertura para el $2019-1$, un $66,73 \%$ para el periodo $2019-2$ y un $66,31 \%$ en periodo COVID-19 el cual puede mejorar para finales de año con la matricula del periodo 2020-2. Reflejando problemas para acceder al beneficio por el puntaje del SISBEN, un sistema que debe ser revisado debido a que impide el ingreso y permanencia de estudiantes en los programas de educación superior.

Gráfico 5

Cantidad total estudiantes matriculados vs beneficiados

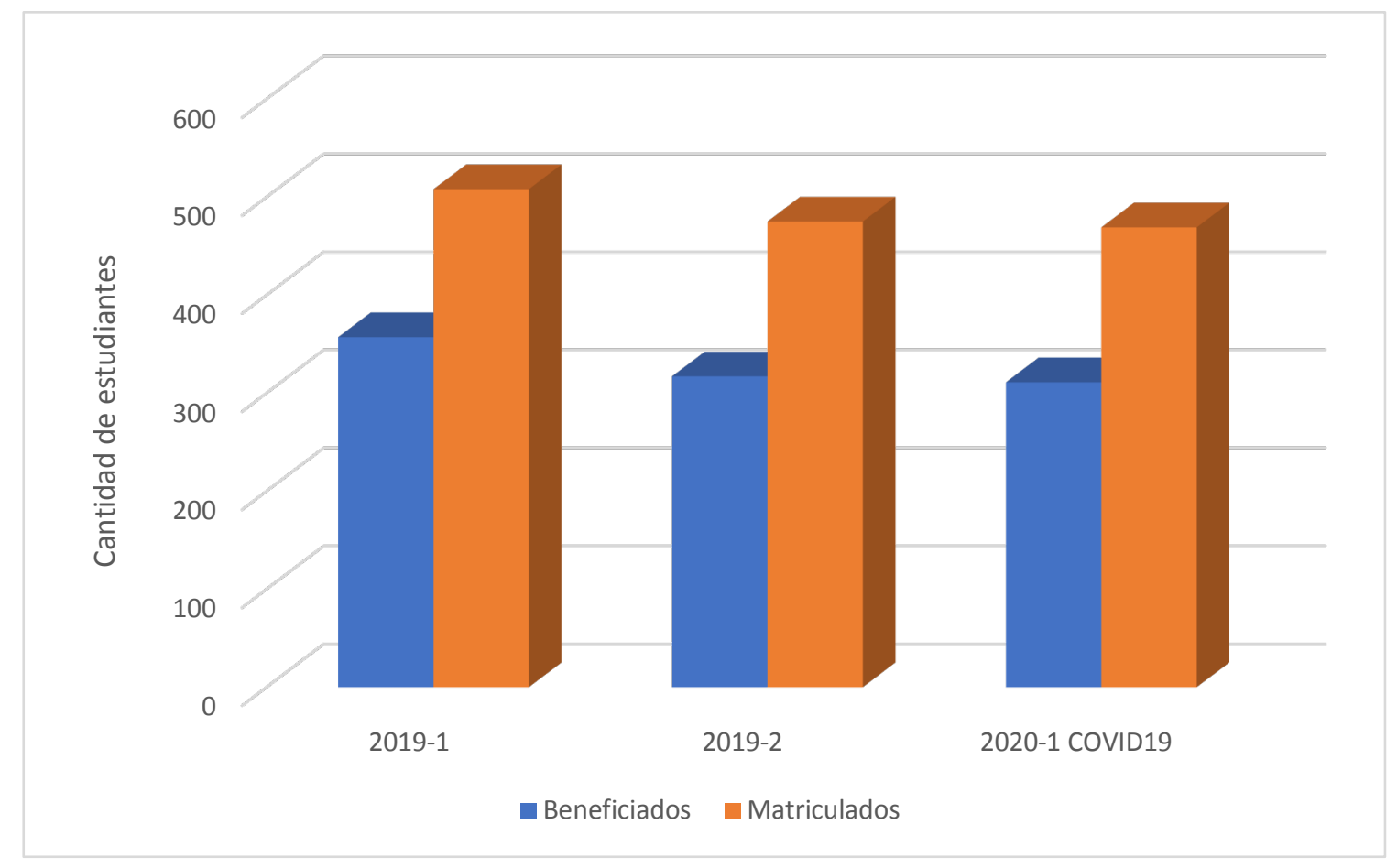

Fuente: elaboración propia

\subsection{Rendimiento académico}

La forma de evaluación en la UFPSO es cuantitativa de 0.0 a 5.0, un estudiante se encuentra condicional cuando su nota es menor a 3.1, activo y no condicional cuando su nota es igual o mayor a 3.1, e inactivo cuando se ha retirado o cancelado semestre. En la Gráfica 6, 93.99\% de estudiantes beneficiados por Generación E se mantienen activos y no condicionados. Al analizar año por año se encuentra que los mejores estudiantes son los que ingresaron cuando se creó el programa, y en tiempos de COVID-19 se ha visto una disminución de estudiantes en situación de incondicionalidad, mejoran sus notas y promedio académico, pero existe un incremento en deserción estudiantil; sin embargo, es debido a la cuarentena y los problemas de su lugar de vivienda, donde no cuentan con un buen acceso a internet, herramienta primordial para continuar con los labores académicas de forma virtual.

El rendimiento académico de los estudiante beneficiados que no tienen condicionalidad es del 93,39 y los estudiantes ingresados en años anteriores y que no pertenecen al programa tienen un porcentaje del 85,35\%; demostrando los beneficios de un estudiante al contar con una ayuda antes de ingresar en un programa de la educación superior. 


\section{Gráfico 6}

Rendimiento académico

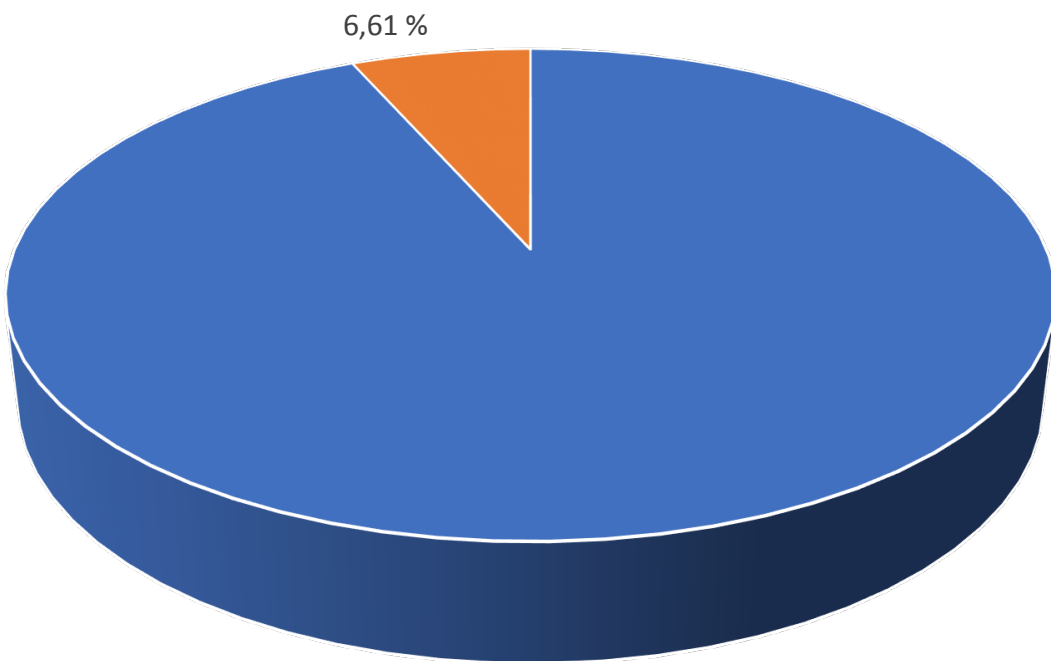

$93,39 \%$

- No condicional $=$ Condicional

Fuente: elaboración propia

\section{Conclusiones}

El programa Generación E es una de las iniciativas del gobierno para cerrar la brecha de desigualdad en la educación superior. Se inició en el primer semestre de 2019 en la UFPSO y muestra que sus beneficios antes y durante el COVID-19 han permitido a los estudiantes mantenerse en la institución, con un rendimiento académico no condicionado del 93,39\%, mostrando una cobertura menor al $78 \%$, lo cual no es lo deseable debido a los recursos disponibles para el programa.

Una de las razones es el puntaje otorgado por el SISBEN, el cual es contrario a lo manifestado por los estudiantes que no han podido ingresar al programa.

La cantidad de estudiantes aunque mínima en deserción mostró un aumento, el cual está directamente relacionado con la falta del servicio eléctrico y la conexión a internet, variables que pueden desaparecer en corto tiempo tan pronto se acabe el aislamiento de alternancia y los estudiantes puedan volver a las aulas de la universidad.

\section{Referencias bibliográficas}

Alonso, G. (2019, marzo). Equidad, para avanzar en la transformación social. Recuperado 9 de octubre de 2020, de https://www.larepublica.co/analisis/gloria-alonso-2793634/equidad-para-avanzar-en-latransformacion-social-2834148

Aranaz Andrés, J. M., Gea Velázquez de Castro, M. T., Vicente-Guijarro, J., Beltrán Peribáñez, J., García Haro, M., Valencia-Martín, J. L., ... Gómez Montero, G. (2020). Mascarillas como equipo de protección individual durante la pandemia de COVID-19: Cómo, cuándo y cuáles deben utilizarse. Journal of Healthcare Quality Research, 35(4), 245-252. https://doi.org/10.1016/j.jhqr.2020.06.001 
ASCUN. (2020). Análisis breve de cifras de educación superior en Colombia | Asociación Colombiana de Universidades. Recuperado 10 de octubre de 2020, de https://www.ascun.org.co/noticias/detalle/analisisbreve-de-cifras-de-educacion-superior-en-colombia

Banco Mundial (2020 septiembre). Deuda y desarrollo: Panorama general [Text/HTML]. Recuperado 9 de octubre de 2020, de World Bank website: https://www.bancomundial.org/es/topic/debt/overview

Carvajal, L. S. M., Vergel, C. S. G., y Ortega, M. V. (2020). Impacto del programa jóvenes en acción en la formación inicial de educadores en matemáticas. Revista Boletín Redipe, 9(7), 165-181. https://doi.org/10.36260/rbr.v9i7.1028

Casas-Rojo, J. M., Antón-Santos, J. M., Millán-Núñez-Cortés, J., Lumbreras-Bermejo, C., Ramos-Rincón, J. M., Roy-Vallejo, E., ... Gómez-Huelgas, R. (2020). Características clínicas de los pacientes hospitalizados con COVID-19 en España: Resultados del Registro SEMI-COVID-19. Revista Clínica Española. https://doi.org/10.1016/j.rce.2020.07.003

Castro Archila, D. P. (2018). Equidad en la distribución de recursos para la educación colombiana. Universidad Santo Tomas. https://doi.org/10.15332/tg.mae.2020.0671

Clavijo, S. (2017). Focalización de subsidios: Estratos, Sisbén y familias en acción. Recuperado 10 de octubre de 2020, de Editorial La República website: https://www.larepublica.co/analisis/sergio-clavijo500041/focalizacion-de-subsidios-estratos-sisben-y-familias-en-accion-2541187

DANE. (2020). Encuesta Pulso Social. Recuperado de https://www.dane.gov.co/index.php/estadisticas-portema/encuesta-pulso-social

DPS. (2020, abril). Manual operativo Programa Jóvenes en Acción. Recuperado de http://centrodedocumentacion.prosperidadsocial.gov.co/2020/JeA/M-GI-TM-1-MANUAL-OPERATIVOJOVENES-EN-ACCION-v8-ABRIL.PDF

Melo-Becerra, L. A., Ramos-Forero, J. E., y Hernández-Santamaría, P. O. (2017). La educación superior en Colombia: Situación actual y análisis de eficiencia <sup/>. Revista Desarrollo y Sociedad, (78), 59-111. https://doi.org/10.13043/dys.78.2

Mineducación. (2018, octubre). Gobierno lanzó 'Generación E', el nuevo programa de educación superior que beneficiará a 336 mil estudiantes de todo el país durante el cuatrienio-Ministerio de Educación Nacional de Colombia. Recuperado 10 de octubre de 2020, de https://www.mineducacion.gov.co/1759/w3-article377779.html?_noredirect=1

Mineducación. (2020, mayo). Nuevas medidas de apoyo al sector educativo anunció hoy el Gobierno Nacional-Ministerio de Educación Nacional de Colombia. Recuperado 10 de octubre de 2020, de https://www.mineducacion.gov.co/1759/w3-article-397798.html?_noredirect=1

MINSALUD. (2020a). Directiva 02 de 2020. Por la cual se declara la emergica sanitaria por causa del coronavirus COVID-19 y se adoptan medidas para hacer frente al virus. Recuperado de https://coronaviruscolombia.gov.co/Covid19/docs/decretos/minsalud/resolucion_385_de_2020_minsalud .pdf

MINSALUD. (2020b, marzo). Resolución número 385 del 12 de marzo de 2020. Declaración de la emergencia sanitaria por causa del Coronavirus. Recuperado de https://coronaviruscolombia.gov.co/Covid19/docs/decretos/minsalud/resolucion_385_de_2020_minsalud .pdf

PAHO. (2020, octubre). PAHO/WHO Health Emergencies-About Us | PAHO/WHO. Recuperado 9 de octubre de 2020, de Pan American Health Organization / World Health Organization website: 
https://www.paho.org/disasters/index.php?option=com_content\&view=article\&id=699:aboutus\&ltemid=918\&lang=en

Rojas, S. E. (2018). ¿Se puede hablar de equidad en el sector educativo colombiano? Revista Científica General José María Córdova, 16(23), 125-143. https://doi.org/10.21830/19006586.286

SAS, E. L. R. (2020). Las movidas universitarias frente al segundo semestre para evitar deserción. Recuperado 10 de octubre de 2020, de https://www.larepublica.co/empresas/conozca-las-movidas-universitarias-decara-al-segundo-semestre-para-evitar-la-desercion-3006661

Esta obra está bajo una Licencia Creative Commons Attribución-NoCommercial 4.0 International

(cc) BY-NC 